for fracture who would warrant further evaluation. Further work to implement and validate these findings in the EMR system would be necessary.

Disclosure of Interest: None declared

DOI: 10.1136/annrheumdis-2017-eular.6572

\section{FRI0573 OSTEOCYTES ARE INVOLVED IN THE PATHOGENESIS OF OSTEOPOROSIS IN CHRONIC CHOLESTASIS. EFFECTS OF BILIRUBIN AND BILE ACIDS ON OSTEOCYTIC CELL LINES}

S. Ruiz-Gaspà, A. Parés, A. Combalia, P. Peris, A. Monegal, N. Guañabens. Liver and Metabolic Bone Diseases Units, CIBERehd-Hospital Clínic, University of Barcelona, Barcelona, Spain

Background: Mechanisms underlying osteoporosis in chronic cholestasis are complex and poorly understood. In this setting, osteoporosis mainly results from low bone formation, related to the effects of retained substances of cholestasis, such as bilirubin and bile acids. Thus, in "in vitro" studies, unconjugated bilirubin and serum from jaundiced patients decrease osteoblast viability, differentiation and mineralization. However, the influence of cholestasis on osteocytes, the most ubiquitous cells of the skeleton, is unknown.

Objectives: The aim of this study was to analyze the direct effects of increased molecules of cholestasis, such as bilirubin (Bil) and lithocholic acid (LCA), and the potential protective effect of ursodeoxycholic acid (UDCA) on the osteocytes. Methods: MLO-Y4 and MLO-A5 osteocyte cell lines treated at different times and concentrations with Bil, LCA and UDCA were used to determine: 1) Viability: WST colorimetric method; 2) Differentiation: quantification of alkaline phosphatase (AP) activity; 3) Mineralization: Alizarin red staining quantification; and 4) Apoptosis: quantification of DNA fragmentation and caspase-3 activity.

Results: LCA $(100 \mu \mathrm{M})$ and Bil $(50 \mu \mathrm{M})$ significantly decreased viability in MLOY4 from 72 hours $(10 \%)$ and 48 hours $(11 \%)$, respectively $(\mathrm{p}<0.01)$, and Bil decreased viability $(49 \%)$ in MLO-A5 from 96 hours $(p<0.01)$. Bil decreased AP activity by $47 \%$ after 96 hours, under conditions of differentiation in MLO-Y4 $(p<0.01)$. There were no effects on AP activity in MLO-A5. After 14 days, Bil was associated with a significant mineralization decrease, as high as $87 \%$, in MLO-A5 $(\mathrm{p}<0.02)$. Moreover, Bil and LCA increased apoptosis in MLO-Y4, determined by DNA fragmentation (242\% and $119 \%$, respectively) and caspase- 3 activity (190\% and $251 \%$, respectively) $(\mathrm{p} \leq 0.01)$ after 24 hours. In contrast, UDCA $(100 \mu \mathrm{M})$ increased viability after 72 hours $(11 \%)$ and decreased the deleterious effects of LCA or Bil $(p \leq 0.02)$. UDCA increased AP activity in MLO-Y4 after 72 hours under growth conditions $(p=0.018)$, and after 24 hours under differentiation conditions $(\mathrm{p} \leq 0.01)$.

Conclusions: Bilirubin and lithocholic acid have damaging effects on osteocytic cells decreasing viability, differentiation and mineralization, and increasing apoptosis, effects that are neutralized by the UDCA. These results indicate that substances retained in cholestasis impair osteocytic functions, and therefore may be involved in the pathogenesis of osteoporosis in cholestatic diseases.

Disclosure of Interest: None declared

DOI: 10.1136/annrheumdis-2017-eular.4711

\section{FRI0574 QUALITY OF LIFE ASSESMENT IN POSTMENOPAUSAL OSTEOPOROSIS}

\section{S. Novkovic ${ }^{1}$, G. Stanojevic ${ }^{2} .{ }^{1}$ Institute of rheumatology; ${ }^{2}$ Health Center} "Zvezdara", Belgrade, Serbia

Objectives: To compair two questionaires:a specific one-Osteoporosis Quality of Life Questionnaire (OQLQ) and a general one-Short Form-36 (SF-36), in women with decreased mineral bone density (BMD) and vertebral fractures, and to estimate which one is more useful for everyday work.

Methods: Cross-section study included 50 menopausal women with osteoporosis (OP), who were assessed with the general questionaire, and all known risk factors for osteoporosis were confirmed. Osteodensitometry scan (DXA) was performed on lumbar spine, and based both on T index value and BMD the subjects were divided in two groups:first grour (women with OP, $n=25$ ), second group (women with fractures of spinal vertebra due to OP,regardless of BMD, $n=25$, and with at least 4 months since the last fracture). The quality of life was assessed by two questionaires:a specific one OQLQ- consists of 30 questions divided into 5 areas,which are evaluated from 1 to 7 (the lower the score the more sever function damage and worse quality of life) and the general questionnaire SF-36-containing 36 questions divided into 8 areas (scores from 0 to 100 , where the lower value is also a poorer quality of life).Questionnaires were converted to get two summarized scales,so as to obtain the total score for:physical (PCS) and mental functions (MCS). The two questionnaires were compared using appropriate statistical methods in SPSS.

Results: There was no significant differencev between groups regarding: mean age (first=63.3 \pm 6.0 , second=64.9 \pm 7.8 ), mean age at the start of the menopause (first $=46.8 \pm 5.3$, second $=48.2 \pm 4.2$ years), mean duration of menopause (first=16.4 \pm 6.9 , second $=17.1 \pm 6.9$ year), but there was statistically significant difference between the number of risk factors for OP (first=1.8 \pm 1.2 , second $=2.8 \pm 0.9, p<0.01)$. Statistically significant between group difference $(p<0.01)$ in mean BMD and T score (first-BMD $0.807 \pm 0.057 \mathrm{gr} / \mathrm{cm}^{2}, T$ scor $-3.12 \pm 0.49 \mathrm{SD}$, second- BMD $0.931 \pm 0.172 \mathrm{gr} / \mathrm{cm}^{2}, T$ score $\left.-2.09 \pm 1.45 \mathrm{SD}\right)$. The following DXA values were measured in the second group:8 patients-normal,6-osteopenia,11- osteoporosis, with average number of fractures 2.6 \pm 0.8 . There was statistically significant between group difference in values of PCS OQLQ questionnaire (first $=2.99,42.7 \%$, second $=2.30,32.8 \%, p<0.01$ ), as well as in values of MCS OQLQ (first $=3.18,45.4 \%$, second $=2.17,31.0 \%, p<0.01$ ). Statistically significant difference was also observed regarding values of PCS SF-36 (first $=42.2,42.2 \%$, second $=32.5,32.5 \%, p<0.01$ ), while regarding MCS SF-36 questionnaire there was no significant between group difference (first=40.2, $40.2 \%$, second $=33.3$, $33.3 \%, p>0.05)$. Mutual comparison of two questionnaires demonstrated correspondence and statistically significant decline in the quality of life in a group with fractures when it comes to PCS and MCS at OQLQ questionnaire and did not record between group difference in MCS SF-36 questionnaire.

Conclusions: It was demonstrated that the specific questionnaire provided more accurate information in all areas of quality of life,with general questionnaire failing to record between group differenc in the field of mental functions.Based on the proven value as an instrument of measurement, its simplicity,accuracy and ease of administration,OQLQ questionnaire is more appropriate for routine use in women with decreasing bone quality and fractures of vertebrae due to osteoporosis.

Disclosure of Interest: None declared

DOI: 10.1136/annrheumdis-2017-eular.3721

\section{FRI0575 SAFETY OF DENOSUMAB IN A MONOCENTRIC COHORT OF KIDNEY TRANSPLANT RECIPIENTS}

S. Doddoli, P. Lafforgue, T. Pham. Bouches Du Rhones, Aix Marseille Unversity, APHM, Marseille, Marseille, France

Background: The safety of Denosumab, a fully human monoclonal antibody against RANKL, which was developed for treatment of osteoporosis and prevention of fractures, remains unclear in kidney transplanted patients.

Objectives: Our aim was to assess its clinical and biological tolerance in this specific population.

Methods: Design: Prospective observational monocentric cohort. Inclusion criteria: kidney transplant recipient who received at least one subcutaneous injections of $60 \mathrm{mg}$ denosumab; age $\geq 18$ years

Safety assessment: the following variables were collected every 6 months: infection, reaction at the injection site, plasmatic parameters of renal function and mineral metabolism (estimated glomerular filtration rate, serum creatinine calcium, 1-25 $[\mathrm{OH}]$ vitamin D, PTH)

Results: Patients were recruited from April 2014 to September 2015. All patients received immunosuppression therapy including prednisolone $\geq 5 \mathrm{mg} / \mathrm{d}$. The main baseline characteristics of the 37 kidney transplant recipients were the following [mean]: male: $41 \%$, age: 60.5 years, BMI: 24,1 , transplantation duration: 7.1 years, osteopenic patients: $36 \%$, osteoporosis patients: $64 \%$, total lumbar spine T-score: -2.04 SD, total hip T-score: $-2.7 \mathrm{SD}$, T-score femoral neck: $0.676 \mathrm{~g} / \mathrm{cm}^{2}$, serum creatinine: $132.8 \mathrm{mmol} / \mathrm{L}$, calcium: $2.33 \mathrm{mmol} / \mathrm{L}, 1-25[\mathrm{OH}]$ vitamin $\mathrm{D}$ $93.5 \mathrm{nmol} / \mathrm{L}, \mathrm{PTH}$ 95: ng/l. All patients were prescribed vitamin D and calcium supplementation.

During the mean 12-month follow-up period, there were no unexpected adverse event $[A E]$ or severe adverse event, no graft failure and no deaths. No patient experienced fracture. Only one patient presented an infectious AE with recurrent cutaneous abscess. Renal function remained stable with no difference in serum creatinine between baseline and 12 months for the majority of the kidney transplant recipients. However, 9 recipients experienced a decrease in renal function with a mean increased in serum creatinine of $32.5 \mathrm{micromol} / \mathrm{L}$ between baseline and 12 months. Serum calcium was stable, no hypocalcaemia was observed. Among patients with normal baseline PTH, two presented hyperparathyroidism during the follow-up period. Among the 11 patients with baseline hyperparathyroidism, 7 had an increased PTH level between baseline and 12 months. None were initiated on cinacalcet.

Conclusions: Our results suggest that denosumab is safe in kidney transplant recipients. We did not observe an increase in the infection rates, nor hypocalcemia. However, several patients experienced a decrease in their renal function or an increased hyperparathyroidism.

Disclosure of Interest: None declared

DOI: 10.1136/annrheumdis-2017-eular.6899

\section{FRI0576 COMPARING TREATMENT INDICATION BY FRAX AND BMD ALONE IN RHEUMATIC PATIENTS ON LONG-TERM GLUCOCORTICOID IN HONG KONG}

S.L. Lau ${ }^{1}$, M.L. Yip ${ }^{2}$, L.-S. Tam ${ }^{1}$, K.L. Lee ${ }^{3}$ on behalf of The Hong Kong GIOP Study Group. ${ }^{1}$ Department of Medicine \& Therapeutics, The Chinese University of Hong Kong: ${ }^{2}$ Department of Medicine and Geriatrics, Kwong Wah Hospital; ${ }^{3}$ Department of Medicine, Pamela Youde Nethersole Eastern Hospital, Hong Kong, Hong Kong

Background: Bone mineral density (BMD) may underestimate fracture risk since most patients with fracture had osteopenia or normal BMD. The WHO Fracture Risk Assessment Tool (FRAX) incorporated BMD and clinical risk factors (CRF) to estimate the 10-year probability of major osteoporotic fractures (MOF) and hip fracture. FRAX has been adopted by various guidelines to assess the need for therapeutic intervention. Whether FRAX is superior to BMD in identifying high-risk patients in need of anti-osteoporotic treatment is worth exploring. 
Objectives: To compare the ability of FRAX and BMD alone in discriminating patients with and without fracture, and their effectiveness in identifying antiosteoporotic treatment needs

Methods: 1300 rheumatic disease patients on long-term glucocorticoid were screened for vertebral fracture by radiograph from 7 rheumatology clinics. 220 ambulatory patients ( 110 with vertebral fracture and 110 without vertebral fracture) were recruited and assessed on CRF with FRAX questionnaire, BMD at the lumbar spine, total hip and femoral neck by dual-energy $x$-ray absorptiometry (DXA). The ten-year probability of MOF and hip fracture (FRAX score) with and without femoral neck BMD were calculated with the WHO FRAX tool.

Results: Both groups of patients were matched in gender, disease type and cumulative glucocorticoid dose $(p>0.1)$. Patients in the fracture group were older ( $63 \pm 14$ vs $59 \pm 12$ years, $p<0.01$ ), of lower body mass index $[22.7 \pm 3.5 \mathrm{vs}$ $\left.24 \pm 3.7 \mathrm{~kg} / \mathrm{m}^{2}, p<0.05\right]$, with a higher prevalence of previous fracture [38 $(34.5 \%)$ vs $5(4.5 \%), p<0.01]$, use of vitamin $D[77(70 \%)$ vs $54(49.1 \%), p<0.01]$ and bisphosphonates [23 (20.9\%) vs $7(6.4 \%), p<0.01]$. A receiver operating characteristic (ROC) curve analysis was performed to determine the ability of FRAX score with or without BMD and BMD alone to discriminate fracture status (Table 1). BMD at the femoral neck and FRAX score with BMD were able to provide adequate discrimination with their area under curve (AUC) $>0.70$. With regards to treatment indication, only $53 / 110(48.2 \%)$ of the patients with vertebral fracture were identified as having osteoporosis (T-score $\leq-2.5$ at hip/spine) by DXA. In contrast, FRAX with BMD were able to identify $71 / 110(64.5 \%)$ of the fracture patients whom treatment criteria were met, with a power of 0.97 and effect size of 0.38 (a medium effect) at the 0.05 level.

Table 1. Area under ROC curve on discriminating fracture

\begin{tabular}{|c|c|c|c|}
\hline & AUC & 95\% Confidence Interval \\
\hline \multicolumn{2}{|c|}{ BMD at Femoral Neck } & 0.709 & $0.640-0.778^{*}$ \\
\hline \multicolumn{2}{|c|}{ BMD of Total Hip } & 0.693 & $0.623-0.763^{*}$ \\
\hline \multicolumn{2}{|c|}{ BMD of Lumbar Spine } & 0.654 & $0.581-0.727^{*}$ \\
\hline \multirow[t]{2}{*}{ FRAX with BMD } & MOF & 0.705 & $0.635-0.775^{*}$ \\
\hline & Hip fracture & 0.720 & $0.651-0.789^{*}$ \\
\hline FRAX without & MOF & 0.681 & $0.610-0.752 *$ \\
\hline BMD & Hip fracture & 0.687 & $0.616-0.758^{*}$ \\
\hline
\end{tabular}

Conclusions: In rheumatic patients on long-term glucocorticoid, FRAX score was more effective in identifying patients with intervention needs than BMD alone. It is suggested that FRAX should be used as a routine assessment to identify patients, even asymptomatic, indicated for anti-osteoporotic treatment.

References:

[1] Aspray, T. J. (2015). Fragility fracture: recent developments in risk assessment. Therapeutic Advances in Musculoskeletal Disease, 7(1), 17-25. http://doi.org/10.1177/1759720X14564562.

[2] Rentero, M. L., Amigo, E., Chozas, N., Fernández Prada, M., Silva-Fernández, L., Abad Hernandez, M. A., ... del Pino-Montes, J. (2015). Prevalence of fractures in women with rheumatoid arthritis and/or systemic lupus erythematosus on chronic glucocorticoid therapy. BMC Musculoskeletal Disorders, 16(1), 300. article. https://doi.org/10.1186/s12891-015-0733-9.

Acknowledgements: We would like to acknowledge the Research Grant Council of Hong Kong for funding support (RGC Ref No.14113714).

Disclosure of Interest: None declared

DOI: 10.1136/annrheumdis-2017-eular.3591

\section{FRI0577 CLINICAL CHARACTERISTICS OF SPONTANEUS FRACTURES IN THE BEDRIDDEN PATIENTS UNINTENTIONALLY CAUSED BY CAREGIVERS}

T. Kashiwagura ${ }^{1}$, Y. Kimura ${ }^{1}$, I. Wakabayashi ${ }^{2}$, M. Fujii ${ }^{1}$, K. Nozaka ${ }^{3}$, N. Miyakoshi ${ }^{3}$, Y. Shimada ${ }^{3} .{ }^{1}$ Department of Orthopedic Surgery; ${ }^{2}$ Department of Rehabilitation Medicine, Akita City Hospital; ${ }^{3}$ Department of Orthopedic Surgery, Akita University Graduate School of Medicine, Akita, Japan

Background: The number of bedridden patients in our super-aging society is increasing. We previously studied fractures occurring in bedridden patients during daily activities with caregiver assistance (dry baths, range of motion exercises, transfer to wheelchair, changing diapers, and so forth) and non-traumatic fractures detected by pain, swelling, subcutaneous bleeding, deformity, and so on. These minimal trauma or spontaneous fractures were defined as "spontaneus fractures in the bedridden patients unintentionally caused by caregivers". Despite efforts to draw attention to preventing these injuries, the incidence of such fractures has been increasing. Spontaneus fractures caused by caregivers are characterized by the presence of untreated osteoporosis, contracture or spasticity near the lesion, institutionalization or hospitalization, repeated fractures, femur fracture, and other unknown causes.

Objectives: Spontaneus fractures caused by caregivers frequently occur without any noticeable trauma and at present, predicting the risk of occurrence is difficult. This study aimed to explore the usefulness of bone metabolism markers in predicting the risk for spontaneus fractures caused by caregivers.

Methods: Study subjects were selected from a pool of 28 patients ( 3 men; 25 women) with 33 spontaneus fractures caused by caregivers who were treated in our hospital between April 2006 and July 2016. Patients were selected based on the following inclusion criteria: no renal dysfunction $($ GFR $\geq 60$ ) and those who had undergone measurement of bone metabolism markers. Finally, 12 women with a mean age at onset of $90.6(76-100)$ years were enrolled in the study. The following markers were evaluated: the bone formation markers bone type alkaline phosphatase and intact procollagen type 1 amino-terminal propeptide; the bone resorption markers tartrate-resistant acid phosphatase (TRACP)-5b, urinary deoxypyridinoline (DPD), and serum $\mathrm{N}$-telopeptide; and the bone quality markers undercarboxylated osteocalcin (uCOC), urinary pentosidine (Pen), and homocysteine (Hcy).

Results: Low levels of bone metabolism markers were not observed in these subjects. However, TRACP-5b levels were high in six subjects while urinary DPD levels were abnormally high in all subjects with a mean value of 23.3 (9.2-41.4) $\mathrm{nmol} / \mathrm{mmol}$ creatinine $(\mathrm{Cr})$. With regards to bone quality markers, levels of Hcy and $\mathrm{uCOC}$ were high in two and three subjects, respectively; while Pen levels were abnormally high in ten subjects with a mean value of $0.183(0.0774-0.3115)$ $\mu \mathrm{g} / \mathrm{mg} \mathrm{Cr}$.

Conclusions: The majority of subjects with spontaneus fractures caused by caregivers had untreated osteoporosis and some had repeated fractures, indicating that early treatment of osteoporosis is important. Assessing osteoporosis in bedridden patients is challenging as measurement of bone density is often difficult due to spinal deformity and contracture. Our results suggest that among the bone metabolism markers, DPD and Pen may be useful predictors for the risk of spontaneus fractures caused by caregivers.

\section{References:}

[1] T Kashiwagura et al. Clinical results of spontaneous fractures in the bedridden patients unintentionally caused by caregivers. Orthop Surg Traumatol 56:189193, 2013.

Acknowledgements: The authors would like to thank Y. Sasaki for technical assistance.

Disclosure of Interest: None declared

DOI: 10.1136/annrheumdis-2017-eular.4531

\section{FRI0578 ODONTOID FRACTURES IN THE ELDERLY: AN UNKNOWN OSTEOPOROTIC FRACTURE?}

L.L. Natella ${ }^{1}$, N. Bronsard ${ }^{2}$, J. Allia ${ }^{2}$, L. Hekayem ${ }^{3}$, L. Euller-Ziegler ${ }^{1}$, F. De Peretti $^{2}, \underline{V}$. Breuil ${ }^{1}$. ${ }^{1}$ Rheumatology/University of Nice, Rheumatology/University of Nice; ${ }^{2}$ Department of Orthopedic Surgery; ${ }^{3}$ Emergency Room Department, University of Nice, nice, France

Background: Current WHO definition of osteoporosis excludes cervical fractures. However, in atraumatic odontoid fractures, mainly reported by orthopedic surgeons, bone status has not been described yet [1].

Objectives: To investigate bone status in elderly patients sustaining a low energy odontoid fracture.

Methods: We conducted a prospective study from January 2016 to January 2017 in patients $>65$ years old, hospitalized in Nice University hospital for low energy odontoid fracture. An evaluation of bone status was proposed within 3 months after fracture event. Evaluation included demographic data, clinical risk factors of osteoporosis, bone mineral density (BMD) at spine and hip and vertebral fracture assessment (VFA) by dual X-ray absorptiometry and serum analysis to detect secondary osteoporosis.

Results: 38 patients were hospitalized for odontoid fracture: 8 patients $<65$ years always after a major trauma (mean age $37.1 \pm 14.5 \mathrm{y}$ ) and 30 patients $\geq 65$ years including 3 after a high energy impact. 27 odontoid fractures followed a low energy impact: 18 women and 9 men, mean age 83.8 y. $( \pm 10.7) .8$ patients died before bone status assessment ( 5 men and 3 women), 6 died during hospitalization with a mean delay of 3.5 days $( \pm 1.87)$ and 2 after discharge ( 1 month and 5 month). 3 patients refused bone status evaluation, 5 were lost to follow-up and 1 is awaiting evaluation. Finally 10 patients had bone status evaluation, all women, mean age 84.2 y. $( \pm 8.9)$. None had parental history of hip fracture, 1 had an early menopause, 1 received aromatase inhibitors for breast cancer and 2 had a history of steroid therapy ( $>3$ months). 3 patients had previously received hormone replacement therapy, 1 received bisphosphonate for 5 years and 4 had calcium + vitamin D supplements. Lumbar spine mean T-score was $-1.45( \pm 1.08)$, femoral neck: $-2.37( \pm 0.040)$ and total hip: $-1.99( \pm 0.6)$. VFA analysis revealed 4 unknown vertebral fractures. The table summarizes population bone status: 8 patients out of 10 fulfilled diagnostic criteria of osteoporosis, including 6 with previous fractures. 2 patients with T-score > -1.DS didn't have hip BMD assessment because of bilateral hip replacement but had previous major osteoporotic fractures. No

\begin{tabular}{|l|l|l|l|}
\hline \multicolumn{1}{|c|}{ Lowest T-score } & No fracture (n) & $\begin{array}{c}\text { Previous minor } \\
\text { osteoporotic fracture }(\mathrm{n})\end{array}$ & Previous major osteoporotic fracture $(\mathrm{n})$ \\
\hline T-score $>-1$ DS & 0 & 0 & $\begin{array}{l}2 \\
\text { (1 patient. } 2 \text { femoral neck fracture, } \\
1 \text { patient: pelvic and humeral fractures. } \\
\text { Both patients had vertebral fracture on VFA })\end{array}$ \\
\hline-1 DS $>$ T-score $2-2.5$ & 1 & $1(1$ wrist) & $\begin{array}{l}2 \\
(1 \text { patient humeral and vertebral fracture on } \\
\text { VFA, } \\
1 \text { patient. } 5 \text { ribs fractures })\end{array}$ \\
\hline T-score $<-2.5$ & 2 & $1(2$ wrist fractures) & 1 (vertebral fracture on VFA) \\
\hline
\end{tabular}

